\title{
EARLY DIAGNOSIS OF DIABETIC RETINOPATHY BY THE DETECTION OF MICROANEURYSMS IN FUNDUS IMAGES
}

\author{
Jeline Devadhas ${ }^{1}$ and R. Binisha ${ }^{2}$ \\ ${ }^{1}$ Department of Embedded System Technologies, Anna University, India \\ ${ }^{2}$ Department of Electrical and Electronics Engineering, Anna University, India
}

\begin{abstract}
The detection of microaneurysms is crucial, as it is an early indicator of a complication of prolonged diabetes called Diabetic Retinopathy. In this paper, an automated approach is proposed to detect microaneurysms from retinal fundus images. Firstly, the magenta plane of the input image is extracted and a few preprocessing techniques are carried out. This is followed by the localization and the removal of the optic disk. The threshold value is determined and is optimized using Firefly algorithm. Then top hat transform is applied to detect the microaneurysms. The image quality parameters and the performance parameters were calculated and analyzed on the images of the DIARETDB1 database. The experimental results yielded a sensitivity of $99.80 \%$ before optimization and $100 \%$ after optimization.
\end{abstract}

\author{
Keywords: \\ Diabetic Retinopathy, Microaneurysms, Magenta Plane, Fundus \\ Images, Firefly Algorithm
}

\section{INTRODUCTION}

In the contemporary era, the number of diabetic patients are accelerating each day. Diabetic Retinopathy (DR) is seen as a commonly addressed issue of diabetes that causes blindness. The loss of eyesight in a diabetic patient can be avoided by the early diagnosis of DR. This is achieved by the evaluation of fundus images. Since DR is an escalating pathology, its severity can be determined by the number and the types of lesions occurring on the retina [1]. The existence of microaneurysms (MAs) in the retina is the initial symptom of DR [2]. MAs are the small red dots that occur in a defective retina. Automatic detection of MAs from RGB fundus images plays a significant role in diabetic retinopathy screening at an enormous scale [3] [4]. This lessens the workload of the ophthalmologists and reduces the time for waiting for a single diagnosis report [3] [5].

The first computerized approaches for the segmentation of retinal MAs were described by Lay [6] and Baudoin et al. [7]. These methods determine the maximum of morphological openings with linear structuring elements of different orientations. This step also produces an image from which the structures that are smaller than the structuring element are lost. Therefore, the difference of this image and the original one may be thresholded to obtain MAs. A limitation of this morphological approach by Lay [6] is that the usage of too large structuring elements would result in the detection of tight vessel curvings as possible MAs. However, if the length of the linear structuring element is chosen so that no parts of the vasculature will be incorrectly discovered, true MAs will be lost, since no round structure that is bigger than the structuring element can be distinguished in this way. In [8], Spencer et al. applied preceding illumination and shade correction steps to improve the quality of the fluorescein angiography images before the actual segmentation and detection steps. A Gaussian match filter was used after the bilinear top-hat transformation to enhance MAs. A recursive region growing method produced the segmented MAs. This was the first method that applied an additional classification step, that is, supervised learning based methods are used to filter out spurious candidates. A set of features are calculated for each of the MAs. These features are intended to capture those characteristics that help to discriminate true candidates from false ones, since the first step usually results in a high number of possible candidates.

Oien and Osnes [9] proposed a pre-filtering technique in which the MAs are found by morphological hit and miss transform. Even if this approach was not further investigated, it was the first publication dealing with the detection of MA in color images. Niemeijer et al. [10] detected MA and hemorrhages with a segmentation step based on pixel classification. The strength of this method lies in the detection of larger lesions for which there is no information about size and shape (like hemorrhages). Ram et al. [11] formulated MA screening as a problem of target detection from clutter, where the probability of target occurrence was smaller compared to the clutter. A successive rejection-based strategy is proposed to lower the number of clutter responses to reject specific classes of clutter whereas passing majority of true MAs.

Agurto et al. [12] proposed the multi-scale amplitudemodulation-frequency-modulation method for discriminating between pathological and normal regions in retinal images, where the cumulative distribution functions of the instantaneous amplitude, the instantaneous frequency magnitude, and the relative instantaneous frequency angle from multiple scales are used as texture feature vectors to measure similarity. Priya and Aruna [13] investigated and proposed a computer-based system for identifying normal, non-proliferative diabetic retinopathy (NPDR) and proliferative diabetic retinopathy (PDR) cases. The proposed system uses color fundus images, where the features are extracted from the raw image by using various image processing techniques and fed into a support vector machine (SVM) for classification.

In [25], the authors have put forward a method for the detection of MAs based on its characteristics in image segmentation. Canny edge detection and maximum entropy thresholding were the techniques used. The processing time was 9.53 seconds per image. Four steps were carried out for the detection of MAs in [26]. These steps include preprocessing, candidature extraction, multi feature dictionary and classification. In [27], a method for the detection of MAs in retinal images using ensemble classifier is elaborated. This method detects an initial 
set of candidates using a Gaussian Matched filer and then classifies this set to reduce the number of false positives.

\section{PROPOSED METHOD}

The MA diagnosis holds a critical role in early detection of DR as these dot-like structures are the first noticeable indications of retinopathy in the eye. The proposed system is applicable in diagnosis which is completely automated and saves a lot of time in generating quick medical reports by experts. MAs are tiny structures. Since the occurrence of these features is among relatively larger features such as macula, optic disc, vascular structure, etc. so the larger features are first removed before the MA detection can begin [14]. The block diagram of the proposed method is shown in Fig.1.

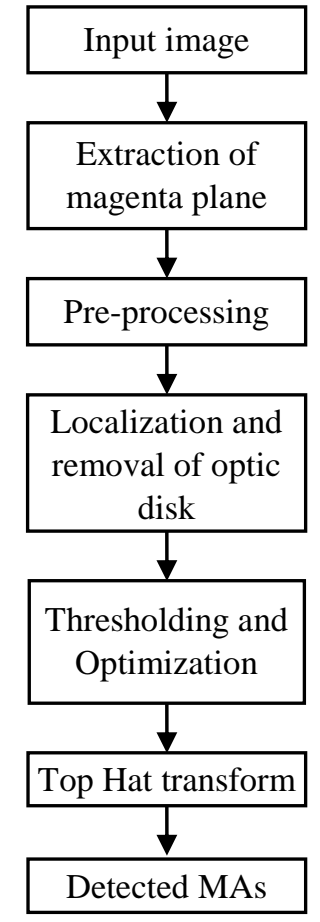

Fig.1 Block diagram of the proposed system

\subsection{INPUT FUNDUS IMAGE}

MAs appear as small reddish isolated patterns of circular shape in color fundus images [15]. They are characterized by their diameter which is always smaller than 125 micro meters. As they are situated on capillaries, and as capillaries are not visible in color fundus images, they appear as isolated patterns that is disconnected from the vascular tree. MAs have typically low contrast and they may be hard to distinguish from noise or pigmentation variations. In [4] the images that were taken for analysis were JPEG compressed and as a result many red lesions that were very small were easily missed. To overcome this problem the proposed system has evaluated PNG images. PNG stands for Portable Network Graphics. It is one of the widely used lossless image compression format. The added advantage of using PNG images is that there is no loss in quality each time it is opened and saved.

\subsection{PREPROCESSING}

Green colored light is generally less absorbed by the retinal layers as compared to the blue colored portion of the spectrum. It is much more than red colored light. The red light as the ability to penetrate deeper into the layers of the retina. The red colored light is less absorbed by the pigments of the interior portion of the eye, and it governs the reflected spectrum. This is the reason why the color fundus images appear reddish. The blood vessels and dark lesions have the highest contrast in the green channel [16]. The red lesions include MAs. It is extracted from the preprocessed image. The red and blue channels are used later to extract color features. In the green channel, MAs and HEs appear as structures with local minimal intensity. In the proposed method the magenta channel is extracted and converted into grayscale. The fundus images often suffer from non-uniform illumination, poor contrast and noise. The preprocessing step aims at attenuating these imperfections. The main goal of the pre-processing stage is the reduction of intensity variations that characterize human retinal images. Preprocessing performs image improvement. The grayscale image is enhanced using Contrast Limited Adaptive Histogram Equalization (CLAHE). The image is filtered using a $3 \times 3$ median filter to remove occasional salt and pepper noise.

\subsection{LOCALIZATION AND REMOVAL OF OPTIC DISK}

The optic disk is a significant source of false positive pixels in the detection of MAs [17] [22] and [23]. Therefore, the removal of the optic disk is an essential step. In [24], an entropy based approach is used to determine the location of the center of the optic disk and refines its position. In the proposed system a structural disk shaped element of size 8 was created and the optic disk was localized. After localization the optic disk was eliminated.

\subsection{THRESHOLDING AND OPTIMIZATION}

The Otsu's thresholding algorithm is a widely used method of segmentation. The algorithm broadly divides the image histograms into two classes, by using a threshold. Assuming that the pixels are categorized into two classes, the algorithm tries to minimize the weighted within class variance $\sigma_{w} i^{2}(t)$ defined by the expression mentioned in Eq.(1). The variable $t$ denotes the threshold which can take values from 0 to 255 .

$$
\sigma_{w} i^{2}(t)=q_{1}(t) \sigma_{2}^{2}(t)+q_{2}(t) \sigma_{2}^{2}(t)
$$

The procedure for computing $\sigma_{w} i^{2}(t)$ is described as follows. Initially the histogram distribution of the image is computed. It then undergoes normalization to ensure it follows a probability distribution. Next, the pixel values are divided into two classes $G_{1}$ and $G_{2}$ by a threshold $t$ using the class probability functions $q_{1}(t)$ and $q_{2}(t)$ as defined in Eq.(2) and Eq.(3).

$$
\begin{gathered}
q_{1}(t)=\sum_{i=1}^{t} P(i) \\
q_{2}(t)=\sum_{i=t+1}^{I} P(i)
\end{gathered}
$$

Class $G_{1}$ represents those pixels with intensity levels $[1, t]$ and class $G_{2}$ represents those pixels with levels in the interval $[t+1, I]$, 
where is typically 255 . The mean value of classes $G_{1}$ and $G_{2}$ are $M_{1}(t)$ and $M_{2}(t)$ are respectively.

$$
\begin{aligned}
& M_{1}(t)=\sum_{i=1}^{t} \frac{i P(i)}{q_{1}(t)} \\
& M_{1}(t)=\sum_{i=t+1}^{I} \frac{i P(i)}{q_{1}(t)}
\end{aligned}
$$

The variances of the two classes $G_{1}$ and $G_{2}$ are $V_{1}(t)$ and $V_{2}(t)$ are computed as follows:

$$
\begin{gathered}
V_{1}(t)=\sum_{i=1}^{t}\left[i-M_{1}(t)\right]^{2} \frac{P(i)}{q_{1}(t)} \\
V_{2}(t)=\sum_{i=t+1}^{I}\left[i-M_{2}(t)\right]^{2} \frac{P(i)}{q_{2}(t)}
\end{gathered}
$$

The proposed system uses thresholding to segment the blood vessels along with the large sized lesions. The type of thresholding used is Otsu's thresholding. The segmented regions are then removed. This step is done to eliminate any false positive values. Optimization algorithms are used in various areas to optimize particular functions and their output. Firefly algorithm has been used in the proposed system provide accurate and efficient results. The firefly algorithm is a nature inspired algorithm. The fireflies produce a type of chemical light from their lower intestines. This bio-luminescence including various flashing patterns and this provides a connection link to the neighboring fireflies. This connection is created to search for nectar and mates. Firefly algorithm is a type of swarm intelligence algorithm in which the behavior and flashing pattern of the fireflies formed its basis [28]. The assumptions for the firefly algorithm are as follows: Every firefly is unisexual. A firefly is attracted to every firefly. Brightness is the key factor of attractiveness. Between two firefly searches agents the less bright one will be attracted towards the brighter one. As the distance increases light intensity decreases which in turn decreases the brightness. The firefly will move randomly when there is no brighter firefly near it. The firefly algorithm has just two basic steps: light intensity variation and attractiveness calculation.

The overall achievement which is inclusive of the exploration time, the speed of convergence, and optimization efficiency of the Firefly Algorithm are dependent on the value function, which monitors optimization search. For a maximizing solution of the problem, the luminance of a firefly is equivalent to the value of the objective function. Luminance is expressed in terms of light intensity. The light intensity $I(r)$ varies with distance $r$ monotonically and exponentially, is given by

$$
I=I_{0} e^{-r \gamma}
$$

where, $I_{0}$ is the initial light intensity and $\gamma$ is the light absorption coefficient. The firefly attractiveness $\beta$ expressed as:

$$
\beta=\beta_{0} e^{-\gamma r^{2}}
$$

where the distance between the two fireflies is denoted by $r$ and $\beta$ is the attractiveness at $r=0$. The Cartesian distance between two fireflies is given as:

$$
r_{i j}=\left\|x_{i}-x_{j}\right\|_{2}=\sqrt{\sum_{k=1}^{d}\left(x_{i, k}-x_{j, k}\right)^{2}}
$$

In the Eq.(10) $x_{i}$ and $x_{j}$ are the positions of the $i^{\text {th }}$ and $j^{\text {th }}$ firefly. During the processing of the firefly algorithm, the less bright firefly $i$ move towards the brighter firefly $j$. The movement is given by:

$$
x_{i}=x_{i}+\beta e^{-\gamma r^{2}}\left(x_{j}-x_{i}\right)+\alpha \varepsilon_{i}
$$

The basic step of the firefly algorithm is the initialization of the firefly population in the algorithm. The size of the population determines the number of solutions. Then each firefly's light intensity is evaluated with its fitness. Distance taken between fireflies is called the Cartesian distance. Light intensity and absorption coefficient are the parameters that define the attractiveness function.

\subsection{TOP HAT TRANSFORM}

Top hat transform can be defined in the following relations. Let $f$ and $s$ be the gray level image and the structuring element respectively. The dilation of $f(x, y)$ is denoted as $f \oplus s$. The erosion of $s(u, v)$ is represented as $f \ominus s$. The two operations are defined as follows:

$$
\begin{aligned}
& f \oplus s=\max (f(x-u, y-v)+s(u, v)) \\
& f \ominus s=\min (f(x+u, y+v)-s(u, v))
\end{aligned}
$$

The opening operation of $f(x, y)$ is defined as:

$$
f \circ s=(f \ominus s) \oplus s
$$

The top hat transform [29] produces the white top hat transform when it undergoes the morphological open operation. The white top hat transform $(W T(x, y))$ is as defined below:

$$
W T(x, y)=f(x, y)-f \circ s(x, y)
$$

The purpose of using Top-hat transform is to extract tiny elements in the images. The MAs are tiny elements in the fundus image. These tiny structures indicate the presence of DR in a fundus image.

\section{EXPERIMENTAL RESULTS}

\subsection{MATERIAL}

The proposed method for MA detection is evaluated on a publicly available benchmark database named Diaretdb1 [20]. This database contains both test images as wells as their corresponding ground truth images. It is one of the most widely used databases primarily designed for different lesion detection algorithms. Among 89 images, 84 contain different signs of DR and the rest are normal. The images are captured with a 50-degree Field of View and a resolution of $1500 \times 1152$. The screenshot of the proposed system's output is shown in Fig.2.

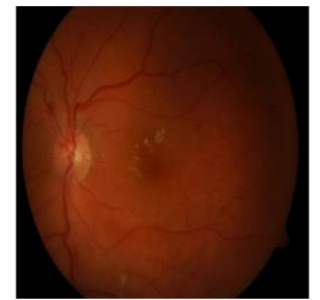

(a)

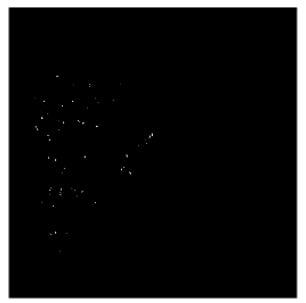

(b)
Fig.2. (a) Input image of the proposed system (b) Output image containing the detected MAs 


\subsection{PERFORMANCE ANALYSIS}

In the preprocessing step, initially the magenta channel is extracted. This is because of a significantly greater value of SSIM in comparison with other channels. The SSIM value is evaluated for two other planes namely cyan and yellow. The Table. 1 shows the comparison of the SSIM index that was obtained for three different channels.

Table.1. SSIM for Different Channels

\begin{tabular}{|c|c|}
\hline Channel & SSIM \\
\hline Cyan & 0.7719 \\
\hline Magenta & 0.9755 \\
\hline Yellow & 0.001 \\
\hline
\end{tabular}

\subsubsection{Image Quality Parameters:}

The image quality parameters were determined for 89 images in the selected database. These parameters include Mean Square Error (MSE), Peak Signal to Noise Ratio (PSNR) and SSIM. Mean Squared Error (MSE) is defined as the square of differences in the pixel values between the corresponding pixels of the two images. The mean squared error (MSE) of $M \times N$ size image is as given below:

$$
M S E=\frac{1}{M \times N} \sum_{i=0}^{M-1} \sum_{j=0}^{N-1}[f(i, j)-g(i, j)]^{2}
$$

where $M$ and $N$ represent the number of rows and columns. The first image pixels are denoted as $f(i, j)$ and the second image is denoted as $g(i, j)$. The Signal to Noise Ratio (SNR) is another image quality parameter. It is defined as follows:

$$
S N R=\frac{\sum_{i=0}^{M-1} \sum_{j=0}^{N-1} g(i, j)^{2}}{\sum_{i=0}^{M-1} \sum_{j=0}^{N-1}[f(i, j)-g(i, j)]^{2}}
$$

The Peak Signal to Noise Ratio (PSNR) is a measure of image quality in which the maximum range of the data type is divided by the MSE. The PSNR value approaches infinity as the MSE approaches 0 . The higher the PSNR value, the better is the quality of the image.

$$
P S N R=10 \log \frac{255^{2}}{M S E}
$$

SSIM (Structural Similarity) index is a popular quality metric for measuring the similarity between two images. The SSIM index is a decimal value between -1 and 1 . The value reaches 1 when both the images are identical. The Eq.(19) defining SSIM is described below:

$$
\operatorname{SSIM}=[l(f, g) \cdot c(f, g) \cdot s(f, g)]
$$

where

$$
\begin{aligned}
& l(f, g)=\frac{2 \mu_{f} \mu_{g}+c_{1}}{\mu_{f}^{2}+\mu_{g}^{2}+c_{1}} \\
& c(f, g)=\frac{2 \sigma_{f} \sigma_{g}+c_{2}}{\sigma_{f}^{2}+\sigma_{g}^{2}+c_{2}}
\end{aligned}
$$

$$
s(f, g)=\frac{\sigma_{f g}+c_{3}}{\sigma_{f} \sigma_{g}+c_{3}}
$$

In the Eq.(19) $l(f, g)$ signifies the luminance factor. $c(f, g)$ and $s(f, g)$ are the contrast and the structure components respectively. $\mu_{f}$ and $\mu_{g}$ are the mean values of samples $f$ and $g$, respectively. $\sigma_{f}^{2}$ and $\sigma_{g}^{2}$ represent the variances of $f$ and $g$, respectively. The covariance is denoted as $\sigma_{f g}$. The Table. 2 shows the image quality parameters that were averaged for 89 images.

Table.2. Image Quality Parameters

\begin{tabular}{|c|c|}
\hline Image quality parameters & Value \\
\hline MSE & 439.411 \\
\hline SNR & 9.8176 \\
\hline PSNR & 21.7018 \\
\hline SSIM & 0.9755 \\
\hline
\end{tabular}

\subsubsection{Performance Evaluation:}

The performance evaluation is done by selecting all the image from the database Diaretdb1 [20]. The performance of the proposed method was tested on a total of 89 retinal images. The performance was tested in terms of sensitivity. Sensitivity is expressed as follows:

$$
\text { Sensitivity }=\frac{T P}{T P+F N} \times 100
$$

where $T P$ stands for the true positive that is the images that are correctly identified as DR images containing MAs, $F N$ stands for the false negatives that is the images that are normal but are identified as images containing MAs. The Table. 3 depicts the calculation involved in determining the sensitivity. The Table.4 shows a comparison of the sensitivity of the proposed system with some existing literature works.

Table.3. Calculation for determining sensitivity

\begin{tabular}{|c|c|c|}
\hline Parameters & $\begin{array}{c}\text { Proposed } \\
\text { System } \\
\text { (Before } \\
\text { Optimization) }\end{array}$ & $\begin{array}{c}\text { Proposed } \\
\text { System } \\
\text { (After } \\
\text { Optimization) }\end{array}$ \\
\hline True Positives $(T P)$ & 83 & 84 \\
\hline False negatives $(F N)$ & 1 & 0 \\
\hline Sensitivity & $98.80 \%$ & $100 \%$ \\
\hline
\end{tabular}

Table.4. Comparison with various methods

\begin{tabular}{|c|c|}
\hline Method & Sensitivity (\%) \\
\hline Quellec et al. [1] & 89.62 \\
\hline Ram et al. [5] & 88.46 \\
\hline Figueiredo et al. [19] & 93.45 \\
\hline Saleh et al. [21] & 84.31 \\
\hline Proposed system (before optimization) & 99.80 \\
\hline Proposed system (after optimization) & 100.00 \\
\hline
\end{tabular}




\subsection{COMPUTATIONAL TIME}

The overall computation time generally depends on the resolution of the image. It is an important parameter that needs to be take into consideration. The proposed system is tested on images with a resolution of $1500 \times 1152$. Using an Intel Core Processor Intel (R) Core (TM) i3 CPU at $2.13 \mathrm{GHz}$, without parallel processing, the execution time of the proposed system written in Matlab R2014b is 5.192seconds without optimization. After optimization, the computational time was 5.2seconds.

\section{CONCLUSION}

An effective method for the detection of MAs is suggested in this paper. This method is fully automated. The experimental results show that the selection of the magenta plane in the preprocessing step is more advantageous due to a higher value of SSIM. The magenta plane is analyzed in comparison with the cyan and the yellow plane of the image.

The results show that the preprocessing step with the extracted magenta plane produces better results. The sensitivity of the proposed system is compared with other existing literature works. It is seen that the proposed system along with optimization yields a high value of sensitivity. The added advantage is low computational time.

As it is completely automated the system would be a boon in medical diagnosis. Hence this concludes that the system is more effective for the early diagnosis of DR by the detection of MAs.

\section{FUTURE WORK}

The proposed system deals with the initial screen of DR by the detection of MAs. The complete grading of DR would require bright lesions such as exudates and cotton wool spots to be considered. In the future work, our proposed system will be adapted to finding bright lesions and dark lesions along with a good classification technique.

\section{REFERENCES}

[1] G. Quellec, M. Lamard, P.M. Josselin, G. Cazuguel, B. Cochener and C. Roux, "Optimal Wavelet Transform for the Detection of Microaneurysms in Retina Photographs", IEEE Transactions on Medical Imaging, Vol. 27, No. 9, pp. 12301241, 2008.

[2] Early Treatment Diabetic Retinopathy Study Research Group, "Early Photocoagulation for Diabetic Retinopathy", Ophthalmology, Vol. 98, No. 5, pp. 766-785, 1991.

[3] M. Abramoff, M. Niemeijer, M. Suttorp-Schulten, M. Viergever, S. Russell and B. Van Ginneken, "Evaluation of a System for Automatic Detection of Diabetic Retinopathy from Color Fundus Photographs in a Large Population of Patients with Diabetes", Diabetes Care, Vol. 31, No. 2, pp. 193-198, 2008.

[4] M. Niemeijer, B. Van Ginneken, J. Staal, M.S.A. SuttorpSchulten and M.D. Abramoff, "Automatic Detection of Red Lesions in Digital Color Fundus Photographs", IEEE Transactions on Medical Imaging, Vol. 24, No. 5, pp. 584 592, 2005.
[5] K. Ram, G.D. Joshi and J. Sivaswamy, "A Successive Clutter Rejection- based Approach for Early Detection of Diabetic Retinopathy", IEEE Transactions on Biomedical Engineering, Vol. 58, No. 3, pp. 664-673, 2011.

[6] B. Lay, "Analyse Automatique Des Images Angiofluorographiques $\mathrm{Au}$ Cours De La Retinopathie Diabetique", Ph.D Dissertation, Centre of Mathematical Morphology, Paris School of Mines, 1983.

[7] C.E. Baudoin, B.J. Lay and J.C. Klein, "Automatic Detection of Microaneurysms in Diabetic Fluorescein Angiographies", Journal of Epidemiology and Public Health, Vol. 32, No. 3-4, pp. 254-261, 1984.

[8] T. Spencer, J.A. Olson, K.C. McHardy, P.F. Sharp and J.V. Forrester, "An Image-Processing Strategy for the Segmentation and Quantification of Microaneurysms in Fluorescein Angiograms of the Ocular Fundus", Computer and Biomedical Research, Vol. 29, No. 4, pp. 284-302, 1996.

[9] G.E. Oien and P. Osnes, "Diabetic Retinopathy: Automatic Detection of Early Symptoms from Retinal Images", Proceedings of International Norwegian Signal Processing Symposium, pp. 135-140, 1995.

[10] M. Niemeijer, B. Van Ginneken, J. Staal, M.S.A. SuttorpSchulten and M.D. Abramoff, "Automatic Detection of Red Lesions in Digital Color Fundus Photographs", IEEE Transactions on Medical Imaging, Vol. 24, No. 5, pp. 584592, 2005.

[11] K. Ram, G.D. Joshi and J. Sivaswamy, "A Successive Clutter Rejection-based Approach for Early Detection of Diabetic Retinopathy", IEEE Transactions on Biomedical Engineering, Vol. 58, No. 3, pp. 664-673, 2011.

[12] C. Agurto, V. Murray, E. Barriga, S. Murillo, M. Pattichis, H. Davis, S. Russell, Michael Abramoff and P. Soliz, "Multiscale AM-FM Methods for Diabetic Retinopathy Lesion Detection", IEEE Transactions on Medical Imaging, Vol. 29, No. 2, pp. 502-512, 2010.

[13] R. Priya and P. Aruna, "Review of Automated Diagnosis of Diabetic Retinopathy using the Support Vector Machine", International Journal of Applied Engineering Research, Vol. 1, No. 4, pp. 844-863, 2011.

[14] B. Li and H.K. Li, "Automated Analysis of Diabetic Retinopathy Images: Principles, Recent Developments, and Emerging Trends", Current Diabetes Reports, Vol. 13, No. 9, pp. 453-459, 2013.

[15] Pascale Massin and Ali Erginay, "Diabetic Retinopathy", Elsevier, 2000.

[16] T. Walter, P. Massin, A. Erginay, R. Ordonez, C. Jeulin and J.C. Klein, "Automatic Detection of Microaneurysms in Color Fundus Images", Medical Image Analysis, Vol. 11, No. 6, pp. 555-566, 2007.

[17] Istvan Lazar, Andras Hajdu "Retinal Microaneurysm Detection through Local Rotating Cross-Section Profile Analysis", IEEE Transactions on Medical Imaging, Vol. 32, No. 2, pp. 400-407, 2013.

[18] R.J. Winder, et al., "Algorithms for Digital Image Processing in Diabetic Retinopathy", Computerized Medical Imaging and Graphics, Vol. 33, No. 8, pp. 608-622, 2009.

[19] I. Figueiredo, S. Kumar, C. Oliveira, J. Ramos and B. Engquist, "Automated Lesion Detectors in Retinal Fundus 
Images", Computers in Biology and Medicine, Vol. 66, pp. 47-65, 2015.

[20] Tomi Kauppi, V. Kalesnykiene, J.K. Kamarainen, R. Voutilainen, J. Pietila, H. Kalviainen and H. Uusitalo, "Diaretdb1 Diabetic Retinopathy Database Evaluation Protocol", Available at: http://www.it.lut.fi/project/imageret/diaretdb1/

[21] M.D. Saleh and C. Eswaran, "An Automated DecisionSupport System for Non-Proliferative Diabetic Retinopathy", Computer Methods and Programs in Biomedicine, Vol. 108, No. 1, pp. 186-196, 2012.

[22] L. Seoud, T. Faucon, T. Hurtut, J. Chelbi, F. Cheriet and J.M.P. Langlois, "Automatic Detection of Microaneurysms and Haemorrhages in Fundus Images using Dynamic Shape Features", Proceedings of IEEE $11^{\text {th }}$ International Symposium on Biomedical Imaging, pp. 101-104, 2014.

[23] A.D. Fleming, S. Philip, K.A. Goatman, J.A. Olson and P.F. Sharp, "Automated Microaneurysm Detection using Local Contrast Normalization and Local Vessel Detection", IEEE Transactions on Medical Imaging, Vol. 25, No. 9, pp. 12231232, 2006.

[24] A.M. Mendonca, A. Sousa, L. Mendonca and A. Campilho, "Automatic Localization of the Optic Disc by Combining
Vascular and Intensity Information", Computerized Medical Imaging and Graphics, Vol. 37, No. 5-6, pp. 409-417, 2013.

[25] Syna Sreng, Noppadol Maneerat and Kazuhiko Hamamoto, "Automated Microaneurysms Detection in Fundus Images using Image Segmentation", Proceedings of International Conference of Digital Arts, Media and Technology, pp. 233 237, 2017.

[26] Wei Zhou, Chengdong Wu, Dali Chen, Zhenzhu Wang, Yugen Yi and Wenyou Du, "Automatic Microaneurysms Detection based on Multifeature Fusion Dictionary Learning," Computational and Mathematical Methods in Medicine, Vol. 2017, pp. 1-11, 2017.

[27] M.M. Habib, R.A. Welikala, A. Hoppe, C.G. Owen, A.R. Rudnicka and S.A. Barman, "Detection of Microaneurysms in Retinal Images using an Ensemble Classifier", Informatics in Medicine Unlocked, Vol. 9, pp. 44-57, 2017.

[28] X.S. Yang, "Firefly Algorithm, Stochastic Test Functions and Design Optimisation", International Journal of BioInspired Computation, Vol. 2, No. 2, pp. 78-84, 2010.

[29] Geoff Dougherty, “Medical Image Processing: Techniques and Applications", Springer, 2011. 